\title{
Sevoflurane Improved Seizure Quality in a Treat-resistant Depression Patient Undergoing Electroconvulsive Therapy
}

T. Okamoto' ${ }^{1}$ H. Seki ${ }^{1}$, S. Ideno' ${ }^{1}$ R. Minoshima ${ }^{1}$, B. Yamagata ${ }^{2}$, H. Morisaki ${ }^{1}$

${ }^{1}$ Department of Anesthesiology, Keio University School of Medicine - Tokyo (Japan)

2Department of Neuropsychiatry, Keio University School of Medicine - Tokyo (Japan)

\section{Introduction}

- Intravenous anaesthetics are commonly used in electroconvulsive therapy (ECT).

- We report a case suggesting that sevoflurane is more suitable than intravenous anaesthetics for inducing adequate seizures in ECT.

\section{Case}

- A 78-year-old woman who was admitted to our psychiatric unit because of severe depression. At the time of this admission, her height was 151 $\mathrm{cm}$ and weight was $58 \mathrm{~kg}$.

- Pulse wave ECT under general anaesthesia with thiopental and suxamethonium was introduced at the age of 73 . To maintain the remission, she had to take maintenance ECT 2-4 times a month and acute ECT several times a year had been required.

- Due to elevation of convulsion threshold and development of tolerance to thiopental, the sine-wave ECT (a higher energy-output device) was started from the $108^{\text {th }}$ session

- Anaesthetic agent was changed to propofol from the $125^{\text {th }}$ treatment session and sevoflurane has been used instead of intravenous anaesthetics from the $136^{\text {th }}$ treatment session.

- Under sevoflurane anaesthesia, effective convulsive seizures has been obtained even under lower energy output by pulse wave ECT.

\section{Anaesthetic protocol using sevoflurane}

1. Fill the anaesthesia circuit with $8 \%$ sevoflurane in $100 \%$ oxygen.

2. Put a face mask on the patient to administer sevoflurane.

3. Administer suxamethonium after a loss of response to verbal commands

4. Stop administrating sevoflurane and start mask ventilation.

5. Give electrical stimulus after terminating fasciculation, and when expiratory sevoflurane concentration $\leq 0.5-0.6 \%$.

Compared to intravenous anaesthetics, sevoflurane anaesthesia ...(Table 1)

- The incidence of high-amplitude seizure waves $\longrightarrow$ (Relative Risk (RR): 1.43, 95\% Confidence Interval (CI): $0.95-2.14, p=$ 0.21 , Fischer's exact test)

- The median score of post-ictal suppression $(p=0.03$, Mann Whitney test)

- The duration of the seizure $\rightarrow$ (Prop vs Sev: $47.6 \pm 6.5 \mathrm{sec}$ vs $63.1 \pm 4.1 \mathrm{sec}, p=0.06$, Unpaired t-test)

- The rate of effective convulsive seizure $\uparrow$ (RR: 2.5, 95\% I: 1.17-5.34, $p=0.01$, Fischer's exact test)

\section{Discussion}

- Although inhalation anaesthetics have been reported not to have any advantage over intravenous anaesthetics ${ }^{2}$, we assumed that sevoflurane has several advantages as shown in Table 2.

- Measuring the depth of anaesthesia at the time of electrical stimulation may be particularly important because deep anaesthesia disturbs the achievement of effective convulsion ${ }^{3-5}$.

- Sevoflurane is easy to adjust the depth of anaesthesia by monitoring expiratory anaesthetic concentration. Meanwhile, when using intravenous anaesthetics, anaesthesia depth is difficult to titrate without using electroencephalogram monitoring.

- After using anaesthetic sevoflurane for all ECT procedures in our hospital, we achieved a higher success rate of inducing effective convulsion without any complications.

\section{Conclusion}

Sevoflurane may dramatically improve the anaesthesia method for ECT, especially in patients who require repetitive ECT sessions.
Table 1. Parameters of convulsive seizure before and after changing anaesthetic to sevoflurane

\begin{tabular}{|c|c|c|c|c|c|}
\hline Session & $\begin{array}{c}\text { Anaestethic dose } \\
(\mathrm{mg}, \text { or \%) }\end{array}$ & Symmetry & $\begin{array}{c}\text { Hi-amplitude } \\
\text { spike } \\
(>180 \mu \mathrm{V})\end{array}$ & $\begin{array}{l}\text { Post-ictal } \\
\text { suppression } \\
\text { score } \\
\end{array}$ & $\begin{array}{c}\text { Seizure } \\
\text { duration } \\
\text { (s) }\end{array}$ \\
\hline 124 & Thio, 200 & 0 & $x$ & 0 & 60 \\
\hline 125 & Thio , 200+50, Prop, 40 & 0 & 0 & 1 & 15 \\
\hline 126 & Prop, $50+30$ & 0 & 0 & 1 & 64 \\
\hline 127 & Prop, $60+20+40$ & O & O & 2 & 54 \\
\hline 128 & Prop, $50+30$ & 0 & $x$ & 0 & 72 \\
\hline 130 & Prop $80+30$ & 0 & 0 & 2 & 57 \\
\hline 131 & Prop, 150 & 0 & 0 & 2 & 22 \\
\hline 132 & Prop, 120 & 0 & 0 & 2 & 48 \\
\hline 133 & Prop, $80+20+20$ & 0 & $x$ & 0 & 21 \\
\hline 134 & Prop, 120 & 0 & 0 & 2 & 63 \\
\hline 145 & Sev, 8 & $\mathrm{O}$ & $\mathrm{O}$ & 2 & 34 \\
\hline 146 & Sev, 8 & $\mathrm{O}$ & 0 & 2 & 74 \\
\hline 147 & Sev, 8 & 0 & $\mathrm{O}$ & 2 & 50 \\
\hline 148 & Sev, 8 & 0 & 0 & 2 & 73 \\
\hline 149 & Sev, 8 & 0 & 0 & 2 & 60 \\
\hline 150 & Sev, 8 & 0 & 0 & 2 & 64 \\
\hline 151 & Sev, 8 & 0 & 0 & 2 & 62 \\
\hline 152 & Sev, 8 & 0 & 0 & 2 & 67 \\
\hline 153 & Sev, 8 & 0 & 0 & 2 & 74 \\
\hline 154 & Sev, 8 & 0 & 0 & 2 & 73 \\
\hline
\end{tabular}

- Post-ictal suppression was evaluated by psychiatrists who were not involved in the study based on Azuma's evaluation method ${ }^{1}$ 0-3. 0: the end of the seizure is unclear, 1: the end of the seizure is clear, but the post-ictal suppression is not flat, 2 : the post-ictal suppression is flat but gradually shifting, 3 : the end of the seizure is clear and post-ictal suppression is also flattened.

- Red boldface represents the session when an effective convulsive seizure was obtained. The "effective convulsive seizure" meet all of the following three requirements; (1) high-amplitude waves of bilateral symmetry, (2) at least 2 on post-ictal suppression score, and (3) duration of convulsions of 20 seconds or more.

- Thio: thiopental, Prop: propofol, Sev: sevoflurane

Table 2. Comparison of intravenous anaesthetics and sevoflurane in electroconvulsive therapy

\begin{tabular}{ccc}
\hline & $\begin{array}{c}\text { Intravenous } \\
\text { anaesthetics }\end{array}$ & Sevoflurane \\
\hline $\begin{array}{c}\text { Inter-individual } \\
\text { variations }\end{array}$ & Hard & Easy \\
Anaesthesia depth & Need EEG & Low \\
Tolerance & Yes & Predictable \\
Effect on EEG & Anticonvulsant effect & Inducing epileptiform \\
wave & wave \\
Awakng & Rapid & Rapid \\
Measures for & Difficult to titrate & Easy \\
\hline multiple energization
\end{tabular}

EEG: electroencephalogram

\section{References}

1. Azuma H, et al. Psychiatry Clin Neurosci 2007;61:168-73.

2. Ding Z, et al. Anesth Analg 2002;94:1351-64.

3. Nishihara F, et al. Anaesth Intensive Care. 2004;32:661-5.

4. Ochiai R, et al . Anesth Analg. 2004;98:1030-5.

5. Galvez V, et al. Brain stimul. 2016;9:72-7. 